\title{
Three-Dimensional Characterization of Pores in Ti-6Al-4V Alloy
}

\author{
Márcia Regina Baldissera ${ }^{\mathrm{a} *}$, Paulo Rangel Rios ${ }^{\mathrm{b}}$, \\ Luis Rogério Oliveira Hein ${ }^{c}$, Hugo Ricardo Zschommler Sandim ${ }^{\mathrm{d}}$ \\ anstituto de Ciências Exatas, Universidade Federal de Itajubá - UNIFEI, \\ Av. BPS, 1303, CEP 37500-903, Itajubá, MG, Brazil \\ ${ }^{\mathrm{b}}$ Escola de Engenharia Industrial Metalúrgica de Volta Redonda, \\ Universidade Federal Fluminense - UFF, Av. dos Trabalhadores, 420, \\ CEP 27180-000, Volta Redonda, RJ, Brazil \\ 'Departamento de Materiais e Tecnologia, Faculdade de Engenharia de Guaratinguetá, \\ UNESP - Univ Estadual Paulista, Av. Dr. Ariberto Pereira da Cunha, 333, \\ CEP 12516-410, Guaratinguetá, SP, Brazil \\ ${ }^{\mathrm{d}}$ Departamento de Engenharia de Materiais, Escola de Engenharia de Lorena - EEL, \\ Universidade de São Paulo - USP, Pólo Urbo-Industrial, Gleba AI-6 s/n, \\ CEP 12602-810, Lorena, SP, Brazil
}

Received: December 2, 2010; Revised: February 24, 2011

\begin{abstract}
The direct three-dimensional characterization of opaque materials through serial sectioning makes possible to visualize and better quantify a material microstructure, using classical metallographic techniques coupled with computer-aided reconstruction. Titanium alloys are used as biomaterials for bone implants because of its excellent mechanical properties, biocompatibility and enhanced corrosion resistance. The Ti-6Al-4V alloy (in wt. (\%)) with porous microstructure permits the ingrowths of new-bone tissues improving the fixation bone/implant. This is important to understand connectivity, morphology and spatial distribution of pores in microstructure. The Ti-6Al-4V alloy compacts were produced by powder metallurgy and sintered at three distinct temperatures $\left(1250,1400\right.$ and $\left.1500{ }^{\circ} \mathrm{C}\right)$ to obtain distinct microstructures in terms of residual porosity. The visualization of the reconstructed $3 \mathrm{D}$ microstructure provides a qualitative and quantitative analysis of the porosity of Ti6Al4V alloy (volume fraction and pore morphology).
\end{abstract}

Keywords: $3 D$ characterization, serial sectioning, titanium alloys

\section{Introduction}

The microstructure characterization of opaque materials is carried out by means of traditional metallography where 2-D planar sections are observed. Following, the three-dimensional characteristics of the material are then inferred from classical stereology, a field of science where statistics and geometry-based principles are used to describe the 3-D microstructure from 2-D sections ${ }^{1-3}$. However, important microstructural parameters including geometry, spatial distribution and connectivity of the existing phases cannot be fully described by the observation of 2-D planar sections. In this case, the visualization of the 3-D structure is necessary to fully understand their microstructures and corresponding properties ${ }^{1-5}$. The technique of serial sectioning allows the visualization and quantification of 3-D microstructures by coupling traditional metallography (2-D planar sections) and computational simulation ${ }^{2,3,6,7}$. With the increasing development of computational tools regarding acquisition and further processing of digital images this task becomes easier and faster ${ }^{8}$. Serial sectioning comprises the following steps: suitable sample preparation by mechanical or chemical-mechanical polishing, marking by indentations (Vickers indenter), image acquisition, computer-assisted processing of 2-D images, image alignment, rendering and 3-D reconstruction. An important parameter in serial sectioning is to determine the amount of material necessary to describe the microstructure. In this case, each polishing step has to be designed to remove the same amount of material in order to get a representative volume of the material for further 3-D reconstruction ${ }^{1,6,9}$. The amount of material removed after each polishing step can be calculated from the Vickers indentations using simple geometric relationships. The indentations are also very useful to limit the investigated areas. High loading forces during Vickers testing create deep indentations and large deformation areas around the indentation whereas low loading forces produce shallow indentations. In the latter case, the number of sections marked by a given indentation is small making difficult its further 3-D reconstruction ${ }^{3,5,9}$. During the digital acquisition of images in 2-D planar sections there may occurs changes from one image to another due to the rotation of the sample or by lateral displacement. Therefore, after every polishing step one has to align the image to ease the 3-D reconstruction. This alignment can be performed by using dedicated software enabling an accurate spatial visualization of the microstructure $\mathrm{e}^{3,9,10}$.

The 3-D reconstruction of metallographic sections of powdermetallurgy processed Ti-6Al-4V alloy sintered compacts has been chosen to validate our technique. Titanium alloys like Ti-6Al-4V have excellent mechanical properties, including a low elastic modulus, biocompatibility and good corrosion resistance in several media ${ }^{2,4-6,11,12}$. In a recent paper, Doi et al. ${ }^{13}$ reported the preparation of commercially-pure $\mathrm{Ti}$ and $\mathrm{Ti}-6 \mathrm{Al}-4 \mathrm{~V}$ materials with coarse pores having Young's moduli as low as $10 \mathrm{GPa}$ for samples with pore volume fraction above $50 \%$. Porous coatings on surgical implants may help to minimize this difference and also allows a faster osteointegration ${ }^{14}$. Furthermore, the morphology and the connectivity 
of the pore network play an essential role to ensure a good adherence of the implant to the bone ${ }^{15}$. Not only aspects related to the pore structure can be evaluated using this technique. Other possibilities might include either the 3-D characterization of the shapes of alpha and beta phase platelets in this two-phase alloy or the morphology of the grains in the sintered material.

In the present work, serial sectioning was used to reconstruct and enabling the visualization of porous structures in Ti-6Al-4V alloy. An optical microscope was used to image the planar sections. The sharp contrast provided by pores in a polished surface is very helpful to image the microstructure. The 3-D reconstructions were used to evaluate the effects of the sintering temperature in the morphology and connectivity of pores in powder metallurgy $(\mathrm{P} / \mathrm{M})$ processed Ti-6Al-4V samples.

\section{Experimental}

Samples of Ti-6Al-4V alloy were prepared by powder metallurgy. The starting powder was obtained by the hydride-dehydride (HDH) process from Ti-6Al-4V alloy turnings. About $1.5 \mathrm{~g}$ of -100 mesh Ti-6Al-4V powder was used to produce each compact. Powder batches were cold pressed in a uniaxial die with $10 \mathrm{~mm}$ in diameter. Green compacts had a density corresponding to about $65 \%$ of the theoretical density of the alloy $\left(\rho_{\text {th }}=4.42 \mathrm{~g} \cdot \mathrm{cm}^{-3}\right)$. High-vacuum sintering was carried out at 1250,1400 and $1500^{\circ} \mathrm{C}$ for 2 hours in order to get distinct microstructures in terms of residual porosity. The sintered compacts were cut, mounted in epoxy, ground in SiC paper (240-1200 grit) and polished with a commercial colloidal silica suspension $(0.05 \mu \mathrm{m})$.

The 3-D serial section reconstruction microscopy studies were carried out according to the following steps: i) marking with aid of a Vickers square-based diamond pyramid indenter with a load of $160 \mathrm{gf}$; ii) digital acquisition of the first image in the light microscope (Leitz, Metallovert, brightfield contrast, variable magnifications); iii) polishing, iv) digital acquisition of the stacking sections (repetition of steps iii and iv); v) alignment of the digital images; vi) rendering for visualization and analysis of porous structure. All the digital images were processed and analyzed with aid of the freeware for image analysis NIH Image J. Spacing between two slices were computed from the reducing on diagonals of Vickers indentation marks. Since the angle $(\phi)$ between opposite faces in square-based pyramid is $136^{\circ}$, then the spacing $(\Delta \mathrm{h})$ is computed from the difference between mean values of Vickers diagonals before and after polishing $\left(\bar{D}_{1}-\bar{D}_{2}\right)$ by:

$$
\Delta h=\frac{\bar{D}_{1}-\bar{D}_{2}}{2 \tan \left(\frac{\varphi}{2}\right)} \approx \frac{\bar{D}_{1}-\bar{D}_{2}}{4.95}
$$

The microstructure of each sintered compact of the Ti-6Al-4V alloys was characterized in 3-D after stacking and alignment of 101 consecutive 2-D planar sections. The amount of material removed after each polishing cycle is about $1.5 \mu \mathrm{m}$. Thus, the reconstructed volume for the sample sintered at $1250{ }^{\circ} \mathrm{C}$ is about $380 \times 310 \times 152 \mu \mathrm{m}^{3}$ and about $770 \times 610 \times 152 \mu \mathrm{m}^{3}$ for the compacts sintered at 1400 and $1500{ }^{\circ} \mathrm{C}$. The difference in volume can be explained by the use of distinct magnification during image acquisition. To ease the visualization of the reconstructed microstructure with emphasis on the residual porosity of each compact, only $15 \%$ of the total volume was used in the respective 3-D reconstructions.

\section{Results and Discussion}

Figure 1 shows the 2-D planar sections of the three sintered samples with their respective Vickers indentations marked by circles. In order to get the most accurate set of micrographs from every sample, variable magnifications were used to image their microstructures at the light optical microscope. The alloy sintered at $1250^{\circ} \mathrm{C}$ has a large pore volume fraction. In this case, a magnification of $200 \mathrm{X}$ was used to acquire the digital images. For the samples sintered at higher temperatures, 1400 and $1500^{\circ} \mathrm{C}$, a magnification of $100 \mathrm{X}$ was sufficient to capture details of the pore structure.

Before starting the 3-D reconstruction, the 2-D planar sections were aligned to ensure they do represent the desired volume with aid of freeware NIH Image $\mathrm{J}^{16}$ using the plug-in StackReg. The StackReg plugin was designed by Philippe Thévenaz, from the Biomedical Imaging Group of the École Polytechnique Fédérale de Lausanne ${ }^{17}$. This plug-in is based on an "a posteriori" algorithm ${ }^{18}$, providing the recursive alignment of stacks where each slice image is used as a template for the next one, based on a coarse-to-fine iterative strategy known as "pyramid approach". This algorithm involves a global three-dimensional affine transformation that can be optionally set by user in four types: translation, rigid body, scaled rotation and affine, all of them combined with isomeric scaling. The choice of better global transformation is dependent of imaging conditions, since alignment needs are derived from sampling process. Figure 2 shows one set of six consecutive slices for one example of sintered compacts. The alignment process is finished only when the images are perfectly aligned each other. Notice that the size of the indentations decrease as the slices are imaged and new indentation marks are necessary to ensure the same region is imaged after each polishing cycle. Vickers impressions have regular shapes and known center points, being very useful to check the consistence of alignment quality.

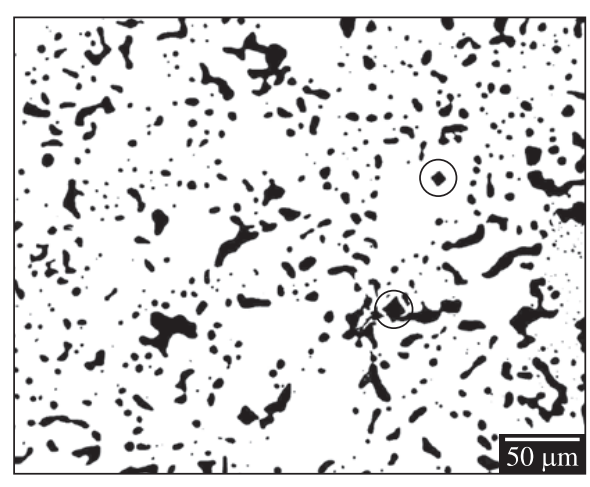

(a)

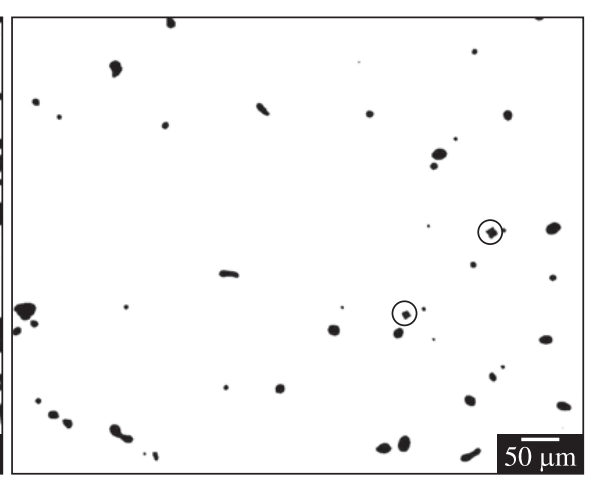

(b)

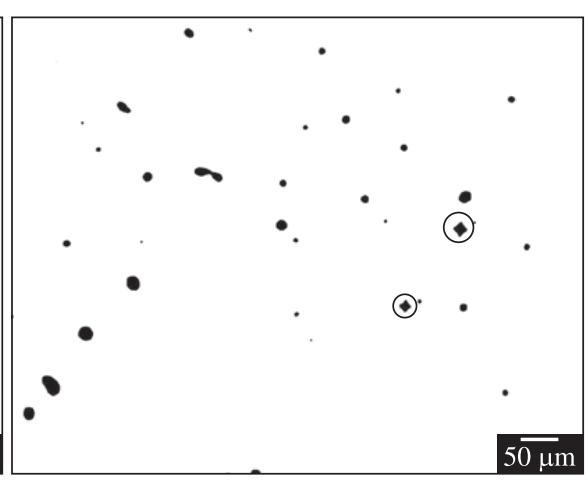

(c)

Figure 1. 2-D planar sections of compacts of Ti-6Al-4V alloy sintered for 2 hours at: a) $1250{ }^{\circ} \mathrm{C}$; b) $1400{ }^{\circ} \mathrm{C}$; and c) $1500{ }^{\circ} \mathrm{C}$. 

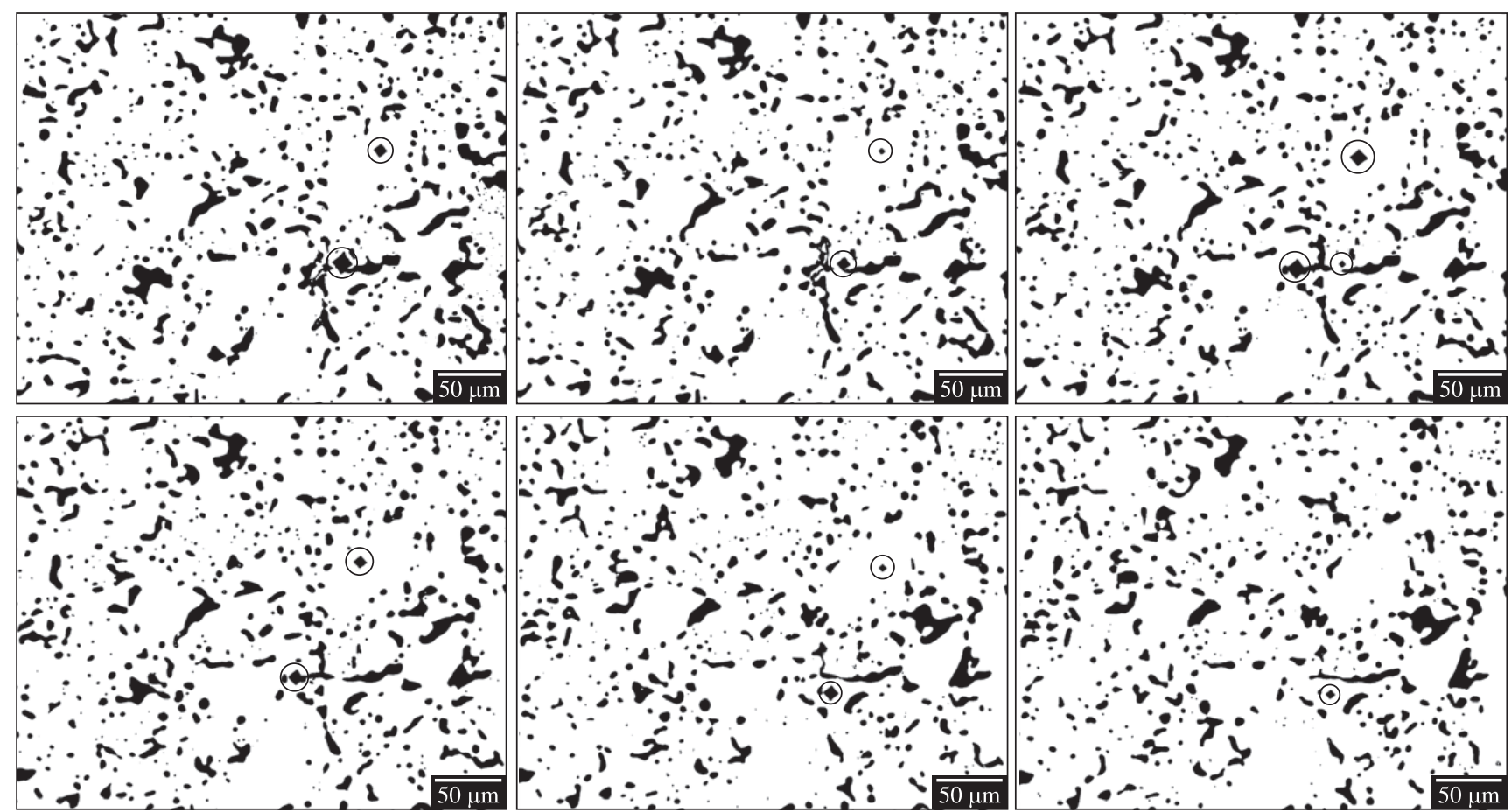

Figure 2. Alignment process in images corresponding to compacts of Ti-6Al-4V alloy sintered for 2 hours at $1250{ }^{\circ} \mathrm{C}$.

The rendering for 3-D reconstruction of aligned stacks was done using the "Volume Viewer" plug-in, developed by K. U. Barthel ${ }^{19}$, setting the "Volume I" display mode to achieve more realistic 3-D scenes. In this display mode, the opacity is controlled by a threshold tool, being possible to visualize sections inside the reconstructed volume. As can be observed in Figures 4, 5 and 6, reconstructed images have enough quality for microstructural analysis, being more interesting due to the public domain character of the software used for stacks alignment and volume rendering.

Figure 3 displays the reconstructed microstructure of the sample sintered at $1250{ }^{\circ} \mathrm{C}$. The 3 -D representation shows a high pore volume fraction. Porosity is also uniform throughout the analyzed volume; however, the accurate description of the pore morphology is blurred or even hidden by the matrix. Figure 4 shows the same volume shown in Figure 3, but without the matrix (removed by the image analysis freeware). In this case, the visualization of the pore structure is eased allowing one to identify all the pores present in this volume. The skeleton-like microstructure consists of elongated and interconnected pores. Contrastingly, in other parts of the microstructure, tiny and isolated spherical closed pores can be seen. It is noticeable that there are very coarse pores in the interior of the imaged volume (marked by arrow) whereas in other parts, smaller and interconnected pores do form channels in the microstructure. These changes in pore structure regarding the morphology, size and spatial distribution of pores in the compact sintered at $1250{ }^{\circ} \mathrm{C}$ is not optimized for applications as coating for surgical implants due to its non-uniformity. A pore volume fraction of $14 \%$ was calculated in this particular reconstructed volume using the freeware Image J. For application as porous structures in surgical implants, much larger porosities are necessary to enable faster osteointegration. Nevertheless, it is worth mentioning that the development of 3-D reconstruction in sintered parts can be extended to other materials enabling the full description of the pore structure in $\mathrm{P} / \mathrm{M}$ processed materials.

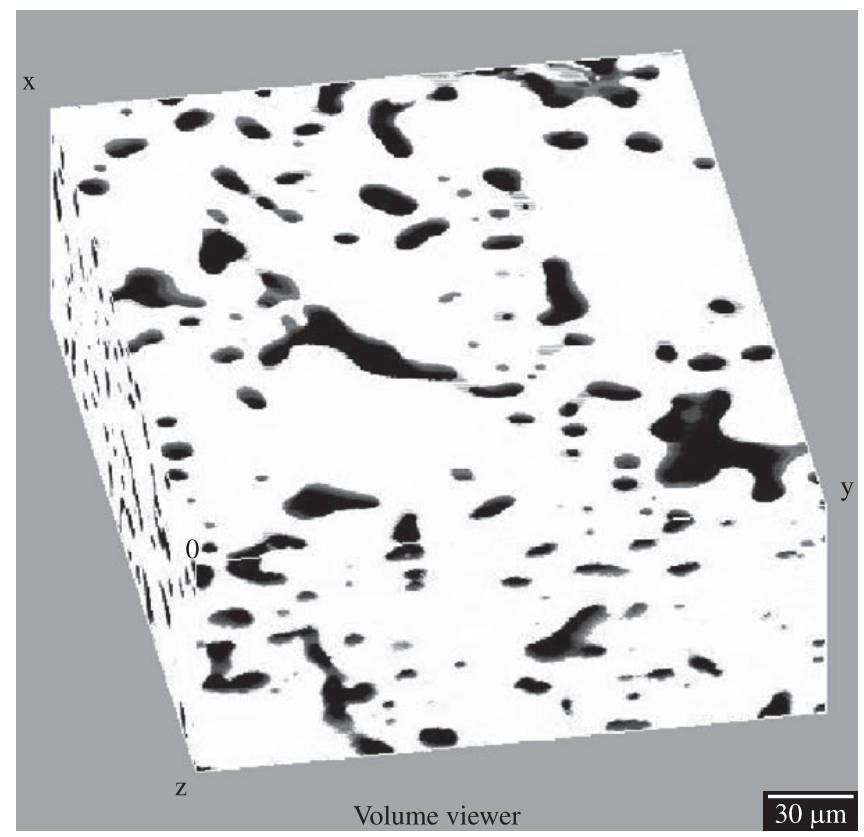

Figure 3. 3-D reconstruction of the microstructure of the Ti-6Al-4V alloy sintered at $1250{ }^{\circ} \mathrm{C}$ showing matrix and pores.

The 3-D reconstruction of the microstructure of the compacts sintered at 1400 and $1500^{\circ} \mathrm{C}$ are shown, respectively, in Figures 5 and 6 . In both cases the pore volume fraction is low and the pore structure is formed by small and round pores. There is no evidence of connectivity among pores, which is expected for such a degree of densification. In the latter stages of densification promoted by solid-state sintering, cylindrical pores tend to break down into isolated and spherical pores. The respective pore volume fractions found in the compacts sintered at 1400 and $1500{ }^{\circ} \mathrm{C}$ are 4 and $3 \%$, respectively. 


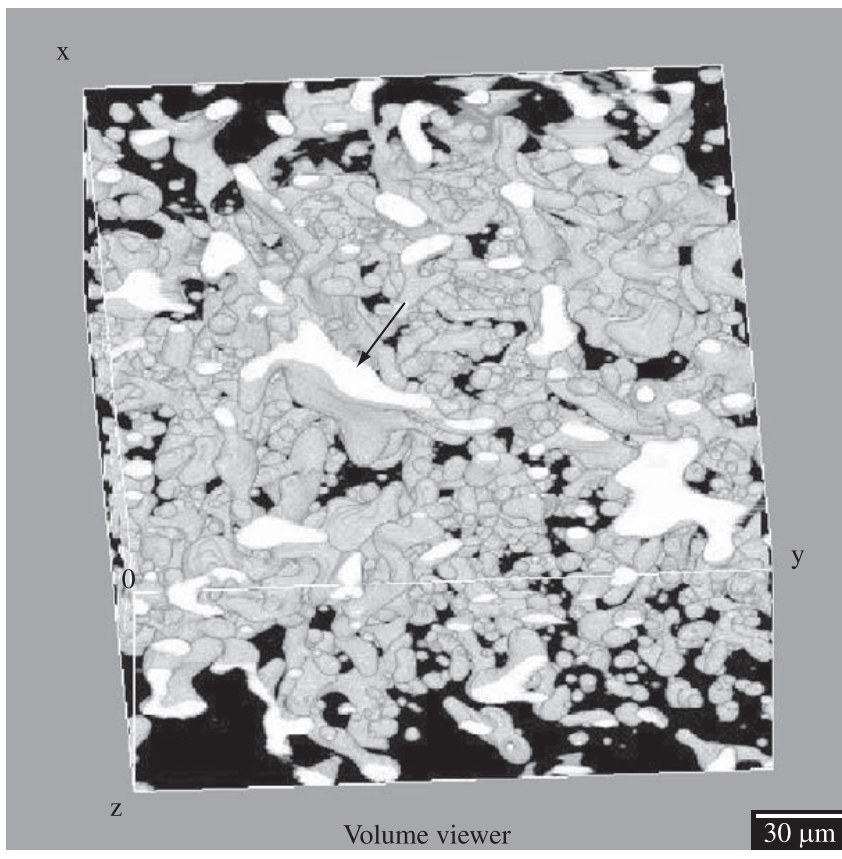

Figure 4. 3-D reconstruction of the microstructure of the Ti-6Al-4V alloy sintered at $1250{ }^{\circ} \mathrm{C}$ showing the pore structure. Matrix was subtracted to ease visualization.

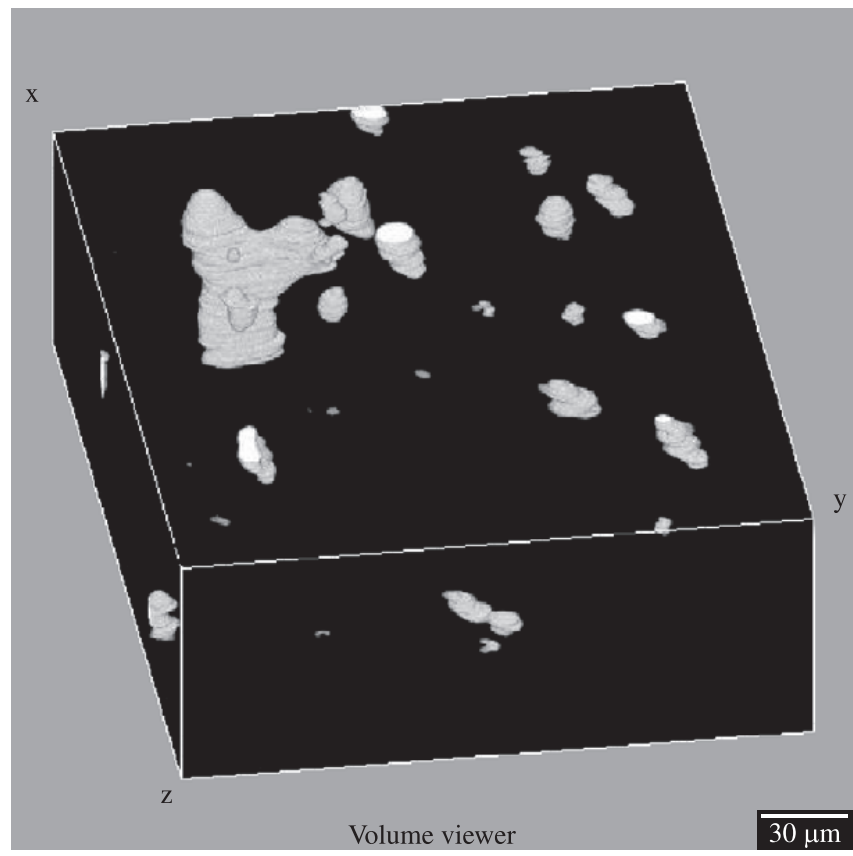

Figure 5. 3-D reconstruction of the microstructure of the Ti-6Al-4V alloy sintered at $1400{ }^{\circ} \mathrm{C}$.

Figure 7 depicts the enlarged view of a small region taken in the compact sintered at $1400{ }^{\circ} \mathrm{C}$ for 2 hours. This detailed image shows that pores are predominantly isolated each other. There is evidence of a single larger connected pore (marked by arrow); however, this seems to be an isolated event in the whole microstructure. Figure 8 also shows an enlarged view of the reconstructed volume found in the sample sintered at $1500{ }^{\circ} \mathrm{C}$. The pore morphology is similar to that one found

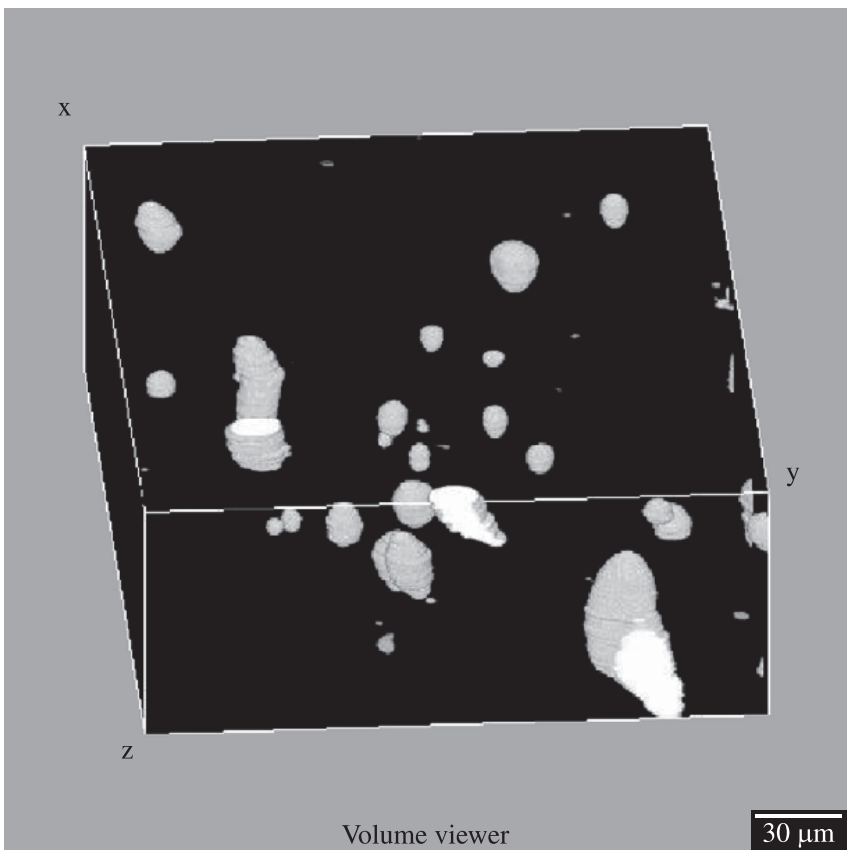

Figure 6. 3-D reconstruction of the microstructure of the Ti-6Al-4V alloy sintered at $1500^{\circ} \mathrm{C}$

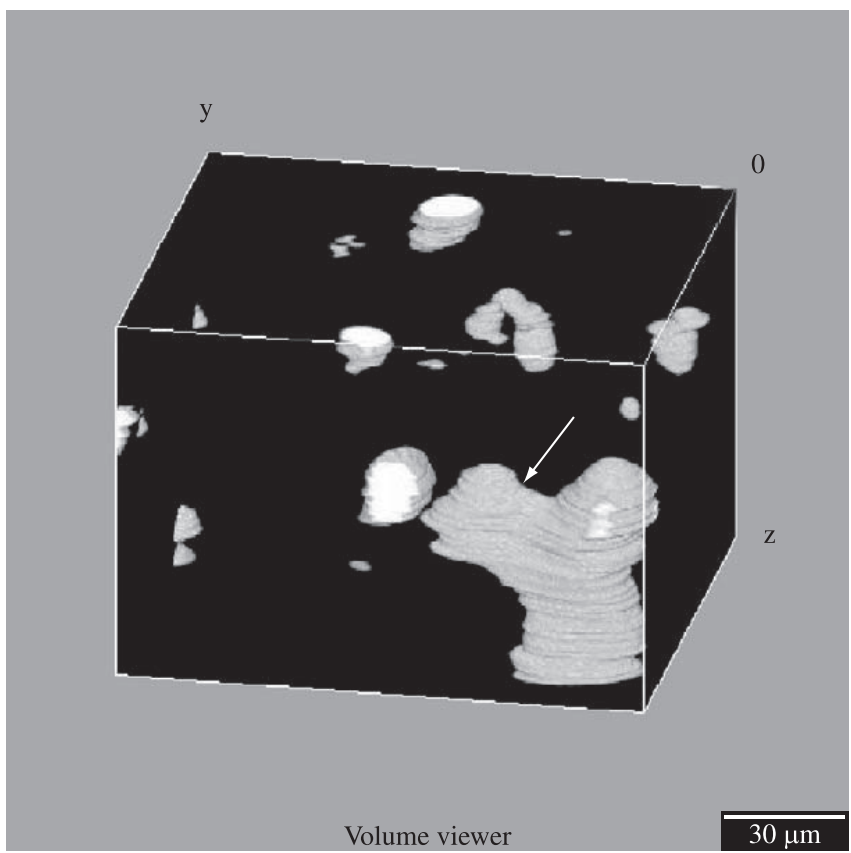

Figure 7. Enlarged view of the 3-D reconstruction in the compact sintered at $1400{ }^{\circ} \mathrm{C}$.

in the sample sintered at $1400{ }^{\circ} \mathrm{C}$, consisting of round closed pores. Pores are rather uniformly spaced each other making connectivity very unlikely. Such a detailed description of the pore morphology in sintered compacts, in particular the connectivity of the pores in the microstructure, can be only be provided by using 3-D reconstruction from serial sections. The conventional 2-D analysis taken from planar sections cannot provide such a level of microstructural understanding. 


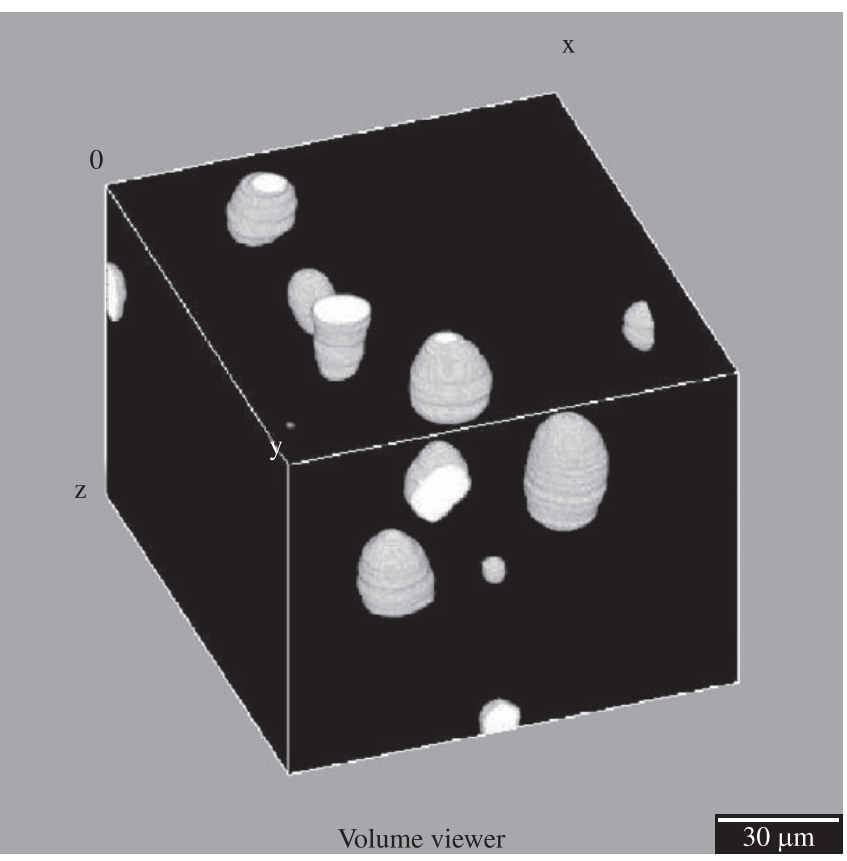

Figure 8. Enlarged view of the 3-D reconstruction showing the pore structure in the compact sintered at $1500{ }^{\circ} \mathrm{C}$.

\section{Conclusions}

Ti-6Al-4V sintered compacts were evaluated in terms of their pore volume fraction, pore morphology, spatial distribution and connectivity using 3-D reconstructions from serial section microscopy. The residual porosity drops with increasing sintering temperatures varying from $14 \%\left(1250^{\circ} \mathrm{C}\right)$ up to $3 \%\left(1500{ }^{\circ} \mathrm{C}\right)$. Round and isolated pores are found in the samples sintered at 1400 and $1500{ }^{\circ} \mathrm{C}$ (latter stages of densification), whereas interconnected pores are found in the microstructure of the compact sintered at $1250{ }^{\circ} \mathrm{C}$ (earlier stages of densification).

3 -D reconstructions were very useful to describe the pore structure in these materials, in particular details of the pore connectivity in the less porous compacts. This simple, low-cost and straightforward routine can be used to investigate other metallic material processed by powder metallurgy.

\section{Acknowledgements}

Authors are deeply acknowledged to CAPES and CNPq (Brazil) for the financial support provided to this work.

\section{References}

1. Kral MV, Mangan MA, Spanos G and Rosenberg RO. Three-dimensional analysis of microstructures. Materials Characterization. 2000; 45:17-23.

2. Krishna BV, Bose $\mathrm{S}$ and Bandyopadhayay A. Low stiffness porous Ti structures for load-bearing implants. Acta Biomaterialia. 2007; 3:997-1006.
3. Lee SG and Gokhale AM. Visualization of three-dimensional pore morphologies in a high-pressure die-cast Mg-Al-RE alloy. Scripta Materialia. 2007; 56:501-504.

4. Lee WH, Park JW, Puleo DA and Kim JY. Surface characteristics of a porous-surfaced Ti-6Al-4V implant fabricated by electro-dischargecompaction. Journal Materials Science. 2000; 35:593-598.

5. Tewari A and Gokhale AM. Estimation of three-dimensional grain size distribution from microstructural serial sections. Materials Characterization. 2000; 44:259-269.

6. Wunsch B and Chawla N. Serial sectioning for 3D visualization and modeling of $\mathrm{SiC}$ particle reinforced aluminum composites. Proceedings of TMS Outstanding Student; 2003. 1-16.

7. Graef M, Kral MV and Hillert M. A Modern 3-D View of an "OId" Pearlite Colony. Proceedings of JOM. 2006; 25-28.

8. Lewis AC, Bingert JF, Rownhorst DJ, Gupta A, Geltmacher AB and Spanos G. Two- and three-dimensional microstructural characterization of a super-austenitic stainless steel. Materials Science and Engineering A. 2006; 418:11-18.

9. Lieberman SI, Gokhale AM and Tamirisakandada S. Reconstruction of three-dimensional microstructures of $\mathrm{TiB}$ whiskers in powder processed Ti-6Al-4V-1B alloys. Materials Characterization. 2007; 58:527-533.

10. Lieberman SI, Gokhale AM and Tamirisakandada S. Reconstruction of three-dimensional microstructures of TiB phase in a powder metallurgy alloy using montage serial sectioning. Scripta Materialia. 2006; 55:63-68.

11. Wen CE, Mabuchi M, Yamada Y, Shimojima K, Chino Y and Asahina T. Processing of biocompatible porous Ti and Mg. Scripta Materialia. 2001; 45:1147-1153.

12. Vasconcellos LMR, Oliveira MV, Graça MLA, Vasconcellos LGO, Carvalho YR and Cairo CAA. Porous Titanium Scaffolds Produced by Powder Metallurgy for Biomedical Applications. Materials Research. 2008; 11:275-280.

13. Doi K, Kokubo T, Fujibayashi S, Takemoto M, Nakamura T, Teraoaka T et al. Mechanical properties of porous titanium and its alloys fabricated by powder sintering for medical use. Proceedings of $17^{\text {th }}$ International Plansee Seminar; 2009.

14. Oh IH, Nomura N, Masahashi N and Hanada S. Mechanical properties of porous titanium compacts prepared by powder sintering. Scripta Materialia. 2003; 49:1197-1202.

15. Otsuki B, Takemoto M, Fujibayashi S and Neo M. Pore throat size and connectivity determine bone and tissue ingrowth into porous implants: three-dimensional micro-CT based structural analyses of porous bioactive titanium implants. Biomaterials. 2006; 27:5892-5900.

16. Rasband WS. ImageJ. US. Bethesda, Maryland, USA: National Institutes of Health; 1997-2008. Available from: http://rsb.info.nih.gov/ij/.

17. Thévenaz, P. StackReg. Switzerland : École Polytechnique Fédérale de Lausanne ; 2009. Available from : http://bigwww.epfl.ch/thevenaz/ stackreg/.

18. Thévenaz P, Ruttimann UE and Unser M. Transactions on image processing; 1998. p. 27-41.

19. Barthel KW. Volume viewer. USA: National Institute of Health, Bethesda; 2009. Available from: http://rsb.info.nih.gov/ij/plugins/volume-viewer.html. 